\title{
Identification of small molecule inhibitors of RANKL and TNF signalling as anti-inflammatory and antiresorptive agents in mice
}

\author{
Emmanuel Coste, ${ }^{1}$ lain R Greig, ${ }^{2}$ Patrick Mollat, ${ }^{3}$ Lorraine Rose, $^{1}$ Mohini Gray, ${ }^{4}$ \\ Stuart H Ralston, ${ }^{1}$ Rob J van 't Hof'
}

\begin{abstract}
Handling editor Tore K Kvien
- Additional material is

published online only. To view please visit the journal online (http://dx.doi.org/10.1136/ annrheumdis-2013-203700).

${ }^{1}$ Molecular Medicine Centre, Institute for Genetics and Molecular Medicine, University of Edinburgh, General Western Hospital, Edinburgh, UK

${ }^{2}$ School of Medical Sciences, Institute of Medical Sciences, University of Aberdeen,

Aberdeen, UK

${ }^{3}$ Galapagos SASU, Romainville, France

${ }^{4} \mathrm{MRC}$ Centre for Inflammation Research, The Queen's Medical Research Institute, University of Edinburgh, Edinburgh, UK
\end{abstract}

\section{Correspondence to}

Dr Rob van 't Hof, Molecular Medicine Centre, Institute for Genetics and Molecular Medicine, University of Edinburgh, General Western Hospital,

Edinburgh EH42XU, UK; rob.vanthof@igmm.ed.ac.uk

Received 28 March 2013 Revised 26 July 2013 Accepted 8 September 2013 Published Online First 4 October 2013

\section{ABSTRACT}

Introduction Inflammatory joint diseases such as rheumatoid arthritis are associated with local bone erosions and systemic bone loss, mediated by increased osteoclastic activity. The receptor activator of nuclear factor (NF) $\kappa B$ ligand (RANKL) plays a key role in mediating inflammation-induced bone loss, whereas tumour necrosis factor (TNF) plays a central role in the inflammatory process. Here we tested whether a recently identified class of small molecule inhibitors of RANKL signalling ( $A B D$ compounds) also affect TNF signalling and whether these compounds inhibit inflammation in an animal model of rheumatoid arthritis.

Methods The inhibitory effects of the ABD compounds on TNF-induced signalling were tested in mouse macrophage cultures by western blotting and in an $\mathrm{NF \kappa B}$ luciferase-reporter cell line. The anti-inflammatory effects of the compounds were tested in the mouse collagen-induced arthritis model of rheumatoid arthritis. Results The ABD compounds ABD328 and ABD345 both inhibited TNF-induced activation of the NFKB pathway and the extracellular signal-regulated kinase (ERK) and Jun kinase (JNK) mitogen activated protein kinases (MAPKs). When tested in the mouse collageninduced arthritis model of rheumatoid arthritis, the compounds suppressed inflammatory arthritis, inhibited joint destruction and prevented systemic bone loss. Furthermore, one of the compounds (ABD328) showed oral activity.

Conclusions Here we describe a novel class of small molecule compounds that inhibit both RANKL- and TNFinduced $\mathrm{NF}_{\kappa} \mathrm{B}$ and MAPK signalling in osteoclasts and macrophages, and inflammation and bone destruction in a mouse model of rheumatoid arthritis. These novel compounds therefore represent a promising new class of treatments for inflammatory diseases, such as rheumatoid arthritis.

\section{INTRODUCTION}

Increased osteoclastic bone resorption plays a major role in the pathogenesis of several diseases such as osteoporosis, rheumatoid arthritis and periodontal disease. Receptor activator of nuclear factor (NF)- $\kappa \mathrm{B}$ (RANK), a member of the tumour necrosis factor (TNF) receptor superfamily (TNFRSF), plays a central role in regulating osteoclast differentiation and function. ${ }^{1}$ When RANK is activated by receptor activator of nuclear factor $\kappa \mathrm{B}$ ligand (RANKL), a signalling complex is recruited to the intracellular domain of the receptor and this triggers activation of several downstream signalling pathways, such as $\mathrm{NF \kappa B}$ and mitogen activated protein kinase (MAPK) pathways, which promote osteoclast differentiation and bone resorption. ${ }^{2}$ Pro-inflammatory cytokines such as TNF also cause $\mathrm{NF} \kappa \mathrm{B}$ and MAPK activation. While activation of the RANK signalling pathway plays a critical role in mediating the osteoclastic bone destruction that accompanies inflammatory arthritis, RANK is not involved in the pathogenesis of joint inflammation, since osteoprotegerin-a decoy receptor for RANKL-inhibits bone destruction, but not joint inflammation in models of inflammatory arthritis. ${ }^{3}$ While TNF plays a critical role in the pathogenesis of inflammation and enhances RANKL-induced osteoclast formation in vitro, ${ }^{4}$ TNF-dependent arthritis is not accompanied by bone destruction in $\mathrm{Fos}^{-/-}$mice which lack osteoclasts. ${ }^{5}$ Moreover, intervention studies have shown that a combination of treatments which block RANKL-induced osteoclast activation and TNF-induced inflammatory signalling is necessary to fully suppress inflammation and to prevent bone destruction in arthritis. ${ }^{6}$ These findings illustrate that RANKL-induced osteoclast activation and TNF-induced pro-inflammatory signalling play differing and complementary roles as mediators of inflammation and bone destruction in inflammatory diseases. Although peptide inhibitors of $\mathrm{NF \kappa B}$ signalling have been shown to suppress inflammation and bone destruction in models of arthritis, ${ }^{7}$ we are unaware of any small molecules which are capable of inhibiting signalling downstream of both the TNF and RANK receptors. We previously identified a series of biphenylcarboxylic acid derivatives which were found to inhibit osteoclastic bone resorption in vitro and ovariectomy-induced bone loss in vivo by causing osteoclast apoptosis. ${ }^{8}$ Previous studies showed that the parent derivative, the butanediol biphenylcarboxylic acid ester (ABD56), acted by inhibiting RANKL-induced phosphorylation of IкB and extracellular signal-regulated kinase (ERK). ${ }^{9}$ Although active, ABD56 is not metabolically stable as the ester bond is easily cleaved. We therefore developed more stable compounds where the ester bond was replaced by a ketone. The stabilised derivatives ABD328 and ABD345 showed higher potency in osteoclast cultures and ABD328 showed oral activity in the ovx mouse model of osteoporosis. ${ }^{10}$ The receptors for TNF and RANK (the receptor for RANKL) are both members of the TNFRSF, and 
activate signalling through recruitment of TNF-receptor associated factor (TRAF) molecules. ${ }^{11} 12$ We therefore studied whether ABD328 and ABD345 inhibit TNF-induced NFKB and MAPK activation and inflammation and bone loss in the collagen-induced arthritis (CIA) mouse model of RA.

\section{MATERIALS AND METHODS}

\section{Materials}

Chemical reagents were obtained from Sigma (Dorset, UK) and culture media were from Gibco (UK). The test compounds ABD328 and ABD345 were synthesised inhouse using standard methods and their identity and purity were confirmed by combustion analysis, mass spectrometry and nuclear magnetic resonance (NMR) as previously described. ${ }^{13}{ }^{14}$ Recombinant human RANKL was produced as described by Idris et al. ${ }^{15}$ All other cytokines were obtained from R\&D Systems (Abingdon, UK).

\section{Cell culture}

Osteoclasts and bone marrow macrophages were generated from bone marrow cells obtained from the long bones of 35 -month-old mice as previously described. ${ }^{16}$ For generation of bone marrow macrophages, the cells were cultured in aminimal essential medium (MEM) supplemented with 10\% foetal calf serum (FCS), antibiotics and recombinant mouse macrophage colony stimulating factor (M-CSF) $(100 \mathrm{ng} / \mathrm{mL})$ for 3 days, the non-adherent cells removed and the macrophages harvested using cell dissociation buffer (Gibco) and scraping and replated at $10^{4}$ cells per well in 96-well plates. Macrophage viability was measured using the Alamar Blue assay as described previously. ${ }^{16}$ For generation of osteoclasts, the M-CSF treated bone marrow macrophages were plated in 96-well plates $\left(10^{4}\right.$ cells/well $)$ in $150 \mu \mathrm{L}$ of $\alpha \mathrm{MEM}$ supplemented with 10\% FCS, antibiotics, M-CSF $(25 \mathrm{ng} / \mathrm{mL})$ and RANKL (100 ng/mL).
A<smiles>O=C(CCCCCO)c1ccc(-c2ccc(Cl)cc2Cl)cc1</smiles>

ABD328<smiles>Cc1cc(C(=O)CCCCCO)ccc1-c1ccc(F)cc1F</smiles>

ABD345
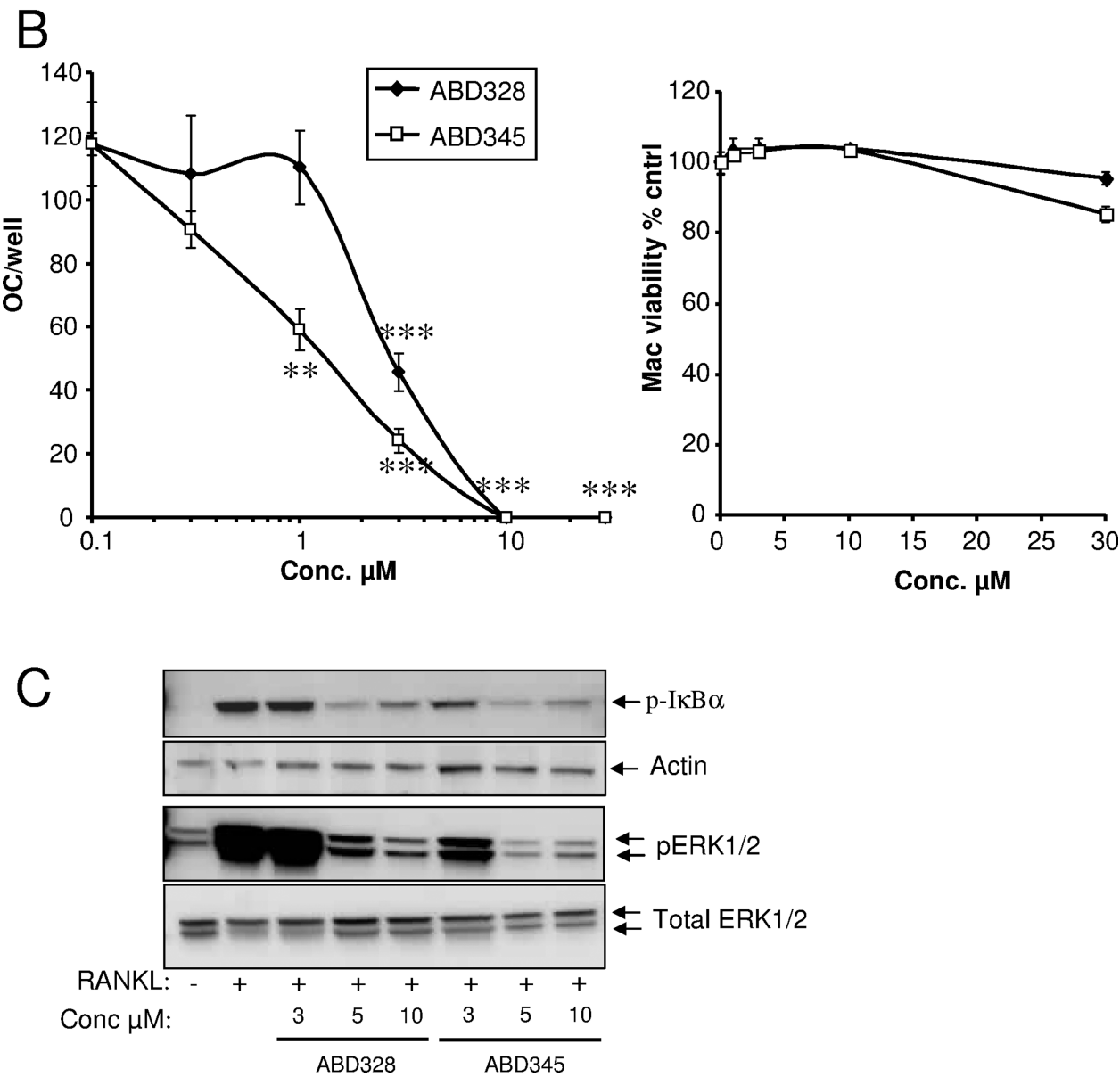

Figure 1 ABD328 and ABD345 are potent inhibitors of osteoclast formation and receptor activator of necrosis factor $\kappa B$ ligand (RANKL)-induced signalling. (A) Structure of the compounds. (B) Osteoclast and bone marrow macrophage cultures were treated with the compounds at the concentrations indicated. ABD328 and ABD345 inhibited osteoclast formation but had no effect on macrophage viability. The results are from a typical experiment $(n=5)$ out of four experiments performed. ${ }^{* *} p<0.01 ;{ }^{* *} p<0.001$. (C) Bone marrow macrophage cultures were serum starved and pretreated with compounds at the concentrations indicated for $1 \mathrm{~h}$. The cultures were stimulated with RANKL (100 ng/mL) for $5 \mathrm{or} 10 \mathrm{~min}$ and IKB (5 min) and extracellular signal-regulated kinase (ERK) $(10 \mathrm{~min})$ phosphorylation analysed by western blotting. 


\section{Intracellular signalling}

Bone marrow macrophages were generated as described above and plated in 6 -well plates at $3 \times 10^{5}$ cells per well in $2 \mathrm{~mL}$ of $\alpha$ MEM supplemented with $10 \%$ FCS, antibiotics and recombinant mouse M-CSF $(100 \mathrm{ng} / \mathrm{mL})$. When the cultures reached semiconfluence, the medium was replaced with serum-free medium and the cells serum starved for $2 \mathrm{~h}$. Next, test compounds or vehicle $(0.1 \%$ DMSO) were added to the media, and the cells cultured for a further $1 \mathrm{~h}$. Cells were stimulated with cytokines for the amount of time indicated, washed in phosphate buffered saline (PBS) and homogenised in lysis buffer (PBS with $0.1 \%(\mathrm{w} / \mathrm{v})$ sodium dodecyl sulfate (SDS), $0.5 \%(\mathrm{w} / \mathrm{v})$ sodium deoxycholate and $2 \%(\mathrm{v} / \mathrm{v})$ protease inhibitor cocktail). The cell lysates were centrifuged at $14000 \mathrm{~g}$ for $10 \mathrm{~min}$ at $4^{\circ} \mathrm{C}$, the supernatants collected and protein concentrations determined using a Bio-Rad protein assay kit. Aliquots of the cell lysates containing $50 \mu \mathrm{g}$ protein per lane were run on a $10 \%$ SDS-acrylamide gel and blotted onto a nylon membrane. The membranes were washed in Tris buffered saline (TBS) and incubated overnight at $4^{\circ} \mathrm{C}$ with the relevant primary antibodies $(1$ : 1000 dilution): actin (Sigma, Poole, UK), phospho-IкBa, total IкBa, phospho-p42/44, total p42/44, phospho-jnk, phospho-p38, phospho-STAT1 and phospho-STAT3 (Cell Signalling Technology, USA). Subsequently, the membranes were washed in TBS and incubated with the appropriate secondary antibodies coupled to horse radish peroxidase (HRP), washed in TBS again, and bands visualised using chemiluminescence (Amersham, UK) on a Syngene GeneGnome imaging system.

\section{Luciferase reporter assays}

The 293/NFkB-luc cell line (Cambridge Scientific, UK) was cultured at $10^{4}$ cells per well in black 96-well plates in $100 \mu \mathrm{L}$ D-MEM supplemented with 10\% FCS and antibiotics for $24 \mathrm{~h}$. Next the medium was replaced with serum free medium with or without test compounds, and the cells cultured for a further $1 \mathrm{~h}$. The cells were stimulated with TNF $\alpha(\mathrm{R} \& \mathrm{D}, 10 \mathrm{ng} / \mathrm{mL})$ for $3 \mathrm{~h}$ and luciferase activity measured using the Promega Steady-Glo system on a Biotek HT platereader.

\section{Collagen-induced arthritis}

Male DBA/1 mice (8-week old; Harlan, UK) were given a $100 \mu \mathrm{L}$ intradermal injection of an emulsion of chicken collagen type II (Sigma, $2 \mathrm{mg} / \mathrm{mL}$ ) in complete Freund's adjuvant supplemented with $1 \mathrm{mg} / \mathrm{mL}$ freeze dried Mycobacterium tuberculosis (Difco) at the base of the tail. Inflammation of the paws was generally first observed after approximately 2 weeks. At this stage, treatment was started by either daily gavage or intraperitoneal injection of $\operatorname{drug}(10 \mathrm{mg} / \mathrm{kg})$ or vehicle (corn oil) for 21 days. The development of joint inflammation was measured by manual scoring using the following scoring system: $0=$ normal, $1=$ erythema or swelling of one or more digits, $2=1$ plus swelling of carpal joints, and $3=$ extensive swelling including hock joints. A total score was calculated based on the sum of counts in all four limbs.

At the end of the experiments, the animals were killed and legs dissected and fixed in phosphate buffered formaldehyde (4\%) for $24 \mathrm{~h}$ and transferred to $70 \%$ alcohol. Paws were
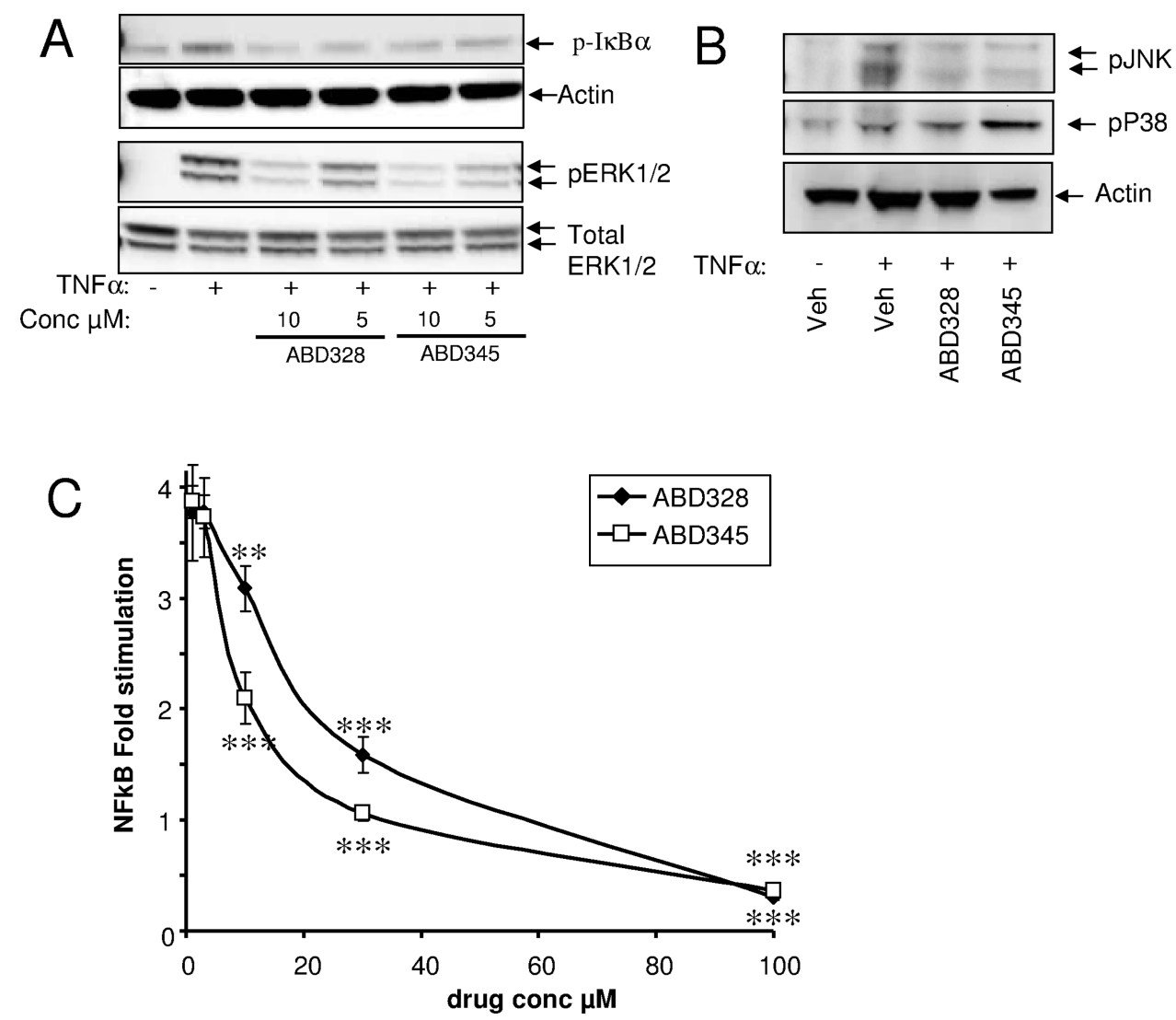

Figure $2 A B D$ compounds inhibit tumour necrosis factor (TNF)-induced signalling. (A and B) Bone marrow macrophage cultures were serum starved and pretreated with compounds at the concentrations indicated (A) or $10 \mu \mathrm{M}$ (B) for $1 \mathrm{~h}$. The cultures were stimulated with TNF (10 ng/mL) for 5 or $10 \mathrm{~min}$ and IKB (5 min) and extracellular signal-regulated kinase (ERK), Jun kinase (JNK) and p38 mitogen activated protein kinase (MAPK) (10 min) phosphorylation analysed by western blotting. (C) 293/NFKB-luc cells were serum starved and pretreated with compounds at the doses indicated for $1 \mathrm{~h}$ before stimulation with TNF $(10 \mathrm{ng} / \mathrm{mL})$ for $3 \mathrm{~h}$ and luciferase activity measured. The results are from a typical experiment $(\mathrm{n}=5)$ out of three experiments performed. ${ }^{* *} p<0.01 ;{ }^{* * *} p<0.001$. 
analysed using a Skyscan $1172 \mu \mathrm{CT}$ system at a resolution of $17 \mu \mathrm{m}\left(60 \mathrm{kV}, 167 \mathrm{~mA}, 0.6^{\circ}\right.$ rotation step). Images were reconstructed using Skyscan NRecon software and visualised using CTVol. After $\mu \mathrm{CT}$ scanning, the samples were dehydrated in ethanol and processed to wax. The samples were cut at $7 \mu \mathrm{m}$ using a microtome, and the sections stained for H\&E. In parallel sections, osteoclasts were identified by TRAcP staining, and the sections counterstained with haematoxylin. Sections were analysed using a Zeiss Axioimager microscope fitted with a QImaging Retiga 4000 camera at $10 \times$ magnification.

For assessment of trabecular bone volume, the proximal tibiae were scanned in a Skyscan 1172 scanner at a resolution of $5 \mu \mathrm{m}$ $\left(60 \mathrm{kV}, 167 \mathrm{~mA}, 0.6^{\circ}\right.$ rotation step). The images were reconstructed as described above and trabecular bone volume was measured in a volume of 200 slices starting $100 \mu \mathrm{m}$ distal of the growth plate.

\section{Statistical analysis}

Statistical analysis was performed using SPSS for Windows. Between-group comparisons were by analysis of variance (ANOVA) with Dunnet's post-test.

\section{RESULTS}

The biphenylcarboxylic acid derivatives ABD328 and ABD345 inhibit both RANKL and TNF-induced signalling

ABD328 and ABD345 were derived from the parent compound ABD56 by replacing the labile ester bond with a stable ketone linkage (figure 1A). Like the parent compound ABD56, ABD328 and ABD345 inhibited osteoclast formation in mouse bone

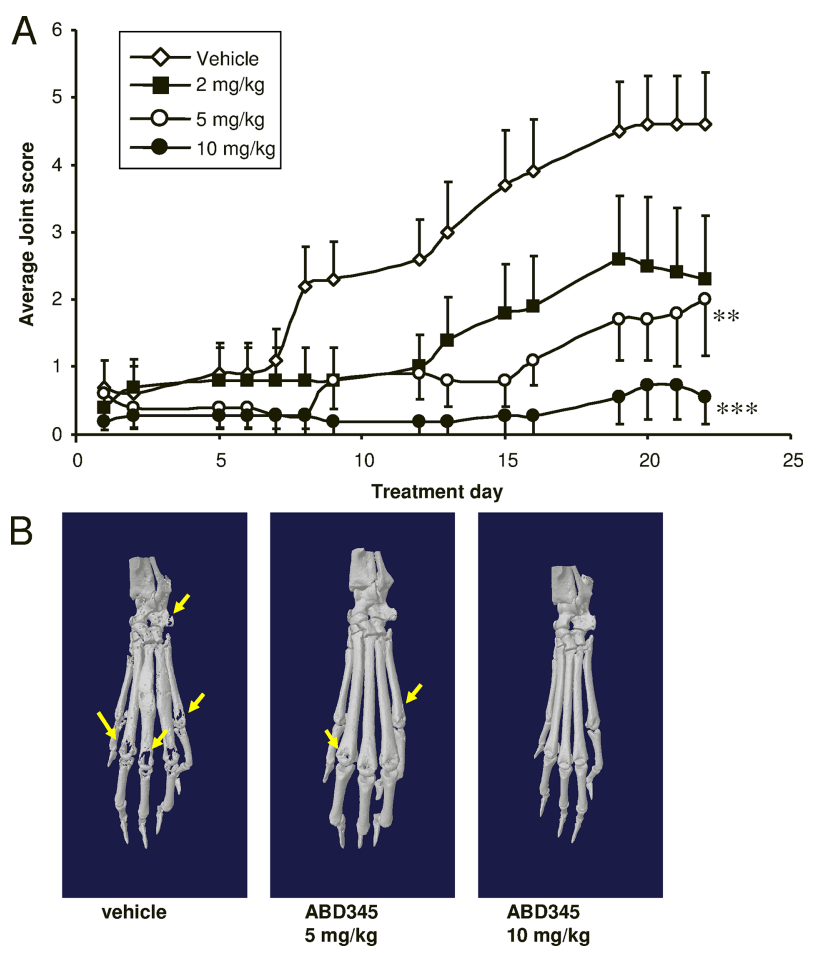

Figure 3 ABD345 dose-dependently inhibits collagen-induced arthritis. (A) Scoring of joint inflammation. Values are averages \pm SEM $(\mathrm{N}=10)$. (B) Examples of joint damage visualised by $\mu \mathrm{CT}$ analysis. Note the severe bone damage in the joints of the vehicle control specimen (yellow arrows), minor damage in the $5 \mathrm{mg} / \mathrm{kg} /$ day specimen and absence of damage in the $10 \mathrm{mg} / \mathrm{kg} /$ day sample. Data were analysed by analysis of variance (ANOVA) using the area under the curve. ${ }^{* *} p<0.01 ;{ }^{* * *} p<0.001$ from vehicle control. marrow cultures without any effects on bone marrow macrophage viability (figure 1B). However, ABD328 and ABD345 were more potent than the parent compound ABD56 (IC50 $8 \mu \mathrm{M}^{8}$ ), and ABD345 (IC50 1.7 $\pm 0.3 \mu \mathrm{M}$ ) was slightly more potent than ABD328 (IC50 2.2 $\pm 0.2 \mu \mathrm{M}$ ). As expected, ABD328 and ABD345 inhibited RANKL-induced phosphorylation of IкB and ERK in M-CSF-dependent bone marrow macrophage cultures (figure 1C). As described above, the receptor for RANKL (RANK) is a member of the TNFR superfamily and activates many of the same signalling cascades as TNF. We therefore tested the effects of ABD328 and ABD345 on TNF-induced signalling. Some signal for phosphorylated IאB was observed in the vehicle control; however, treatment with TNF for 5 min significantly increased the phosphor-IкB signal. ABD328 and ABD345 both inhibited TNF-induced IкB and ERK phosphorylation, and appeared to inhibit TNF- and RANKL-induced signalling with equal potency (figure 2A). As in the osteoclast formation assay, ABD345 appears to be more potent than ABD328. ABD328 and ABD345 also inhibited TNF-induced phosphorylation of Jun kinase (JNK), but not of p38 MAPK (figure 2B). We next studied the effects of the compounds on activation of the downstream $\mathrm{NF}_{\kappa} \mathrm{B}$ transcription factor by TNF using HEK293 cells stably transfected with an NFKB-luciferase reporter construct. Both ABD328 and ABD345 inhibited TNF-induced NFKB activation

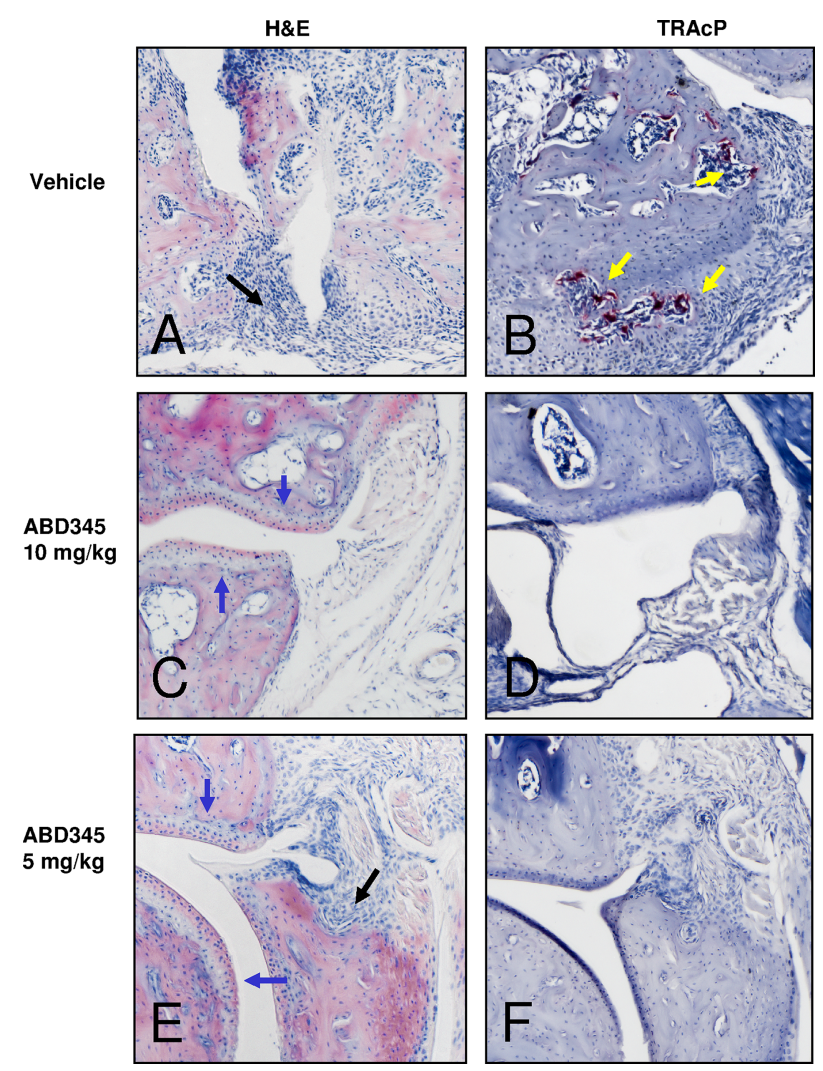

Figure 4 ABD345 inhibits joint inflammation and joint destruction. Examples of histological analysis of hind paws from mice from figure 3. Note the severe joint destruction in the vehicle treated mouse with clear pannus formation (black arrow in A) associated with numerous osteoclasts (yellow arrows in B). No pannus was observed in the $10 \mathrm{mg} / \mathrm{kg} /$ day group (C and D) and cartilage appeared normal (blue arrows, C). Synovial thickening was observed in a small number of joints in the $5 \mathrm{mg} / \mathrm{kg} /$ day group (black arrow, E), but this was not associated with osteoclasts $(F)$ and cartilage appeared normal (blue arrows, E). 
in this assay with ABD345 again more potent than ABD328 (figure 2C).

ABD328 and ABD345 did not inhibit M-CSF- or IFN $\gamma$-induced signalling suggesting that the compounds specifically target the signalling complex of TNFRSF members such as RANK and the TNF receptor (see online supplementary figure S1).

\section{ABD345 prevents collagen-induced arthritis}

As ABD328 and ABD345 inhibited both RANKL- and TNF-induced signalling, these compounds could inhibit inflammation and inflammatory bone loss. As ABD345 was the more potent compound in vitro, we analysed the efficacy of this compound in the collagen-induced arthritis model of inflammatory arthritis. ABD345 was administered by daily intraperitoneal injection and showed a dose-dependent inhibition of collagen-induced arthritis (figure 3). At a dose of $10 \mathrm{mg} / \mathrm{kg} /$ day (given by an intraperitoneal injection), ABD345 almost abolished the development of collagen-induced arthritis as assessed by clinical score (figure 3A). When the paws were analysed by $\mu \mathrm{CT}$, the vehicle control group displayed severe bone erosions near the affected joints (figure 3B), whereas these erosions were virtually absent in the ABD345 treated group. Histological analysis showed typical pannus formation with the presence of large numbers of osteoclasts and severe cartilage and bone destruction in joints from vehicle treated mice (figure 4). In keeping with the inflammation scores and $\mu \mathrm{CT}$ analysis, mice which had been treated with ABD345 at $10 \mathrm{mg} / \mathrm{kg} /$ day showed hardly any inflammatory infiltrate compared with vehicle treated mice and no cartilage or bone destruction (figure 4). At the lower dose of $5 \mathrm{mg} / \mathrm{kg} / \mathrm{day}, \mathrm{ABD} 345$ still showed some

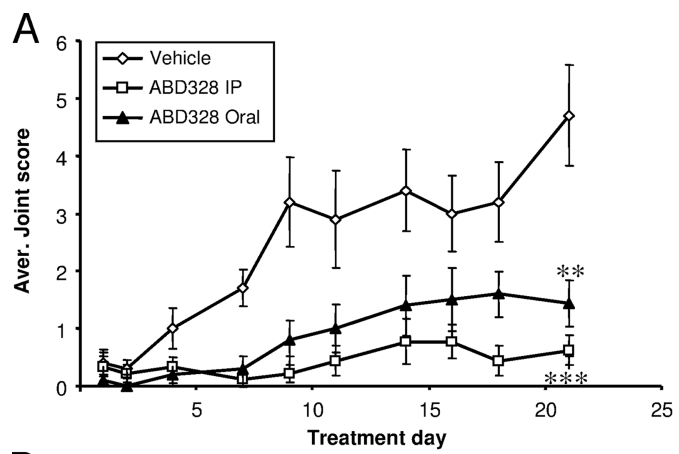

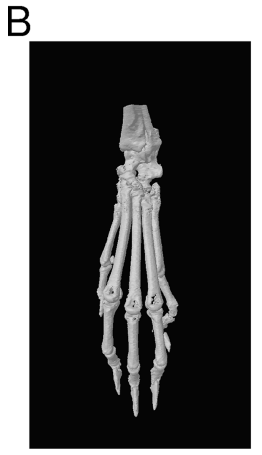

Vehicle

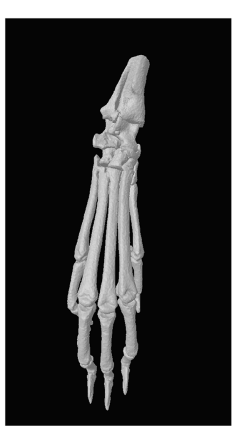

ABD328 IP

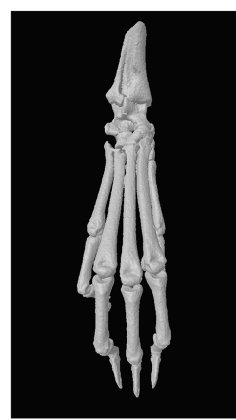

ABD328 Oral
Figure $5 \quad A B D 328$ is an orally active inhibitor of collagen induced arthritis (CIA). (A) Scoring of joint inflammation. Values are averages \pm SEM $(\mathrm{N}=10)$. (B) Examples of joint damage visualised by $\mu \mathrm{CT}$ analysis. Note the joint damage of the vehicle control specimen and absence of damage in the samples from the ABD328 treated mice. Data were analysed by analysis of variance (ANOVA) using the area under the curve. ${ }^{* *} p<0.01 ;{ }^{* * *} p<0.001$ from vehicle control. inhibition of arthritis; however, minor joint damage became apparent in $\mu \mathrm{CT}$ scans, and histological analysis showed some inflammatory infiltrate in some joints, although the cartilage still appeared normal, and we did not observe osteoclasts associated with the inflamed synovium. The dose of $2 \mathrm{mg} / \mathrm{kg} /$ day did not statistically significantly decrease the inflammation scores.

\section{ABD328 is an orally active inhibitor of collagen-induced arthritis}

In a previous study we found that ABD328 prevents ovariectomy-induced bone loss when dosed orally using gavage, ${ }^{10}$ and we therefore tested whether this compound was orally active in the collagen induced arthritis (CIA) model as well. ABD328 given orally at $10 \mathrm{mg} / \mathrm{kg} /$ day significantly reduced progression of the inflammation scores; however, it was not as effective as the same dose administered by intraperitoneal injection (figure 5A). Histology and $\mu \mathrm{CT}$ analysis confirmed that oral ABD328 significantly reduced CIA-induced bone and cartilage destruction (figure 6).

\section{ABD328 and ABD345 inhibit inflammatory bone loss}

The inflammation in the CIA model leads to systemic bone loss in sites not directly affected by the inflammation, such as the knee. Figure 7A shows an almost 25\% decrease in trabecular bone volume in the vehicle treated group. This bone loss is prevented by ABD345 at a dose of $10 \mathrm{mg} / \mathrm{kg} /$ day, while lower doses are not effective (figure 7A). ABD328 prevented trabecular

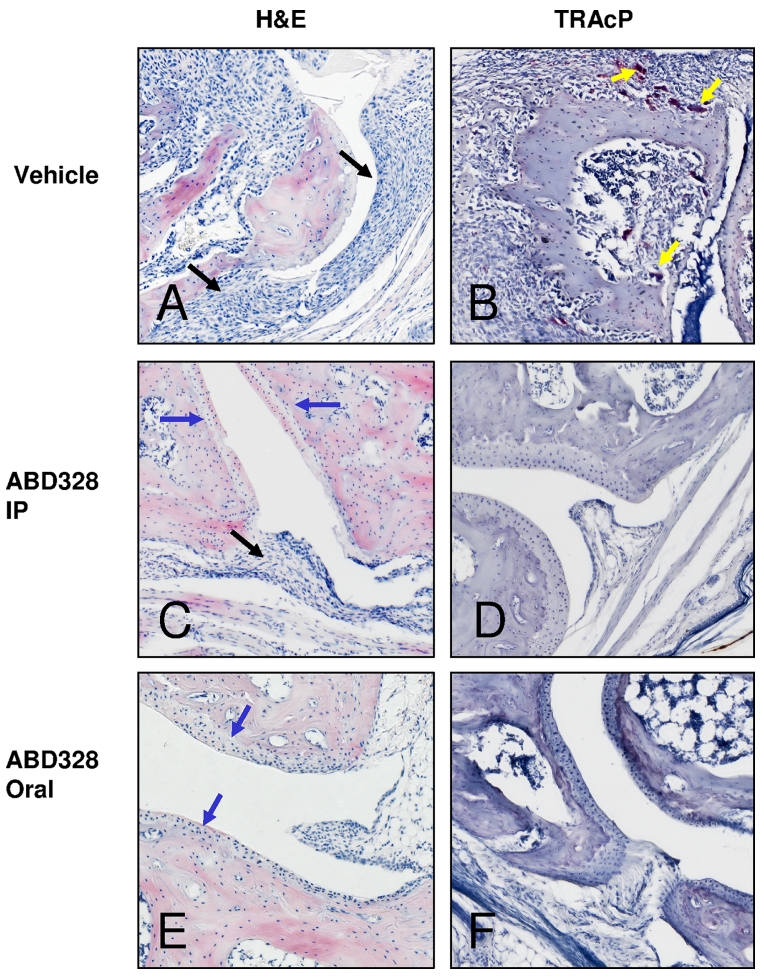

Figure 6 ABD328 prevents joint destruction. Examples of histological analysis of hind paws from mice from figure 5 . ( $A$ and $B$ ) Vehicle control; (C and D) ABD328 $10 \mathrm{mg} / \mathrm{kg} /$ day, by IP injection; ( $E$ and F) ABD328 $10 \mathrm{mg} / \mathrm{kg} / \mathrm{day}$, by gavage. Black arrows indicate synovial thickening, yellow arrows indicate osteoclasts. Some synovial thickening was observed in ABD328 treated animals (C), but this was not associated with increased osteoclasts numbers, and cartilage appeared normal (blue arrows, $\mathrm{C}$ and $\mathrm{E})$. 
bone loss at the same site both by intraperitoneal injection and oral dosing (figure 7B).

\section{DISCUSSION}

In this study, we report the identification of a novel series of small molecule inhibitors of NFKB and MAPK signalling which inhibit signal transduction through both the RANK and TNF receptors. The compounds were effective at inhibiting inflammatory activity in the collagen-induced arthritis mouse model of inflammatory arthritis and the bone loss associated with this arthritis model. Although the important role of NFkB in inflammation is well known, the ERK and JNK MAPK pathways have also been shown to be activated in rheumatoid arthritis and
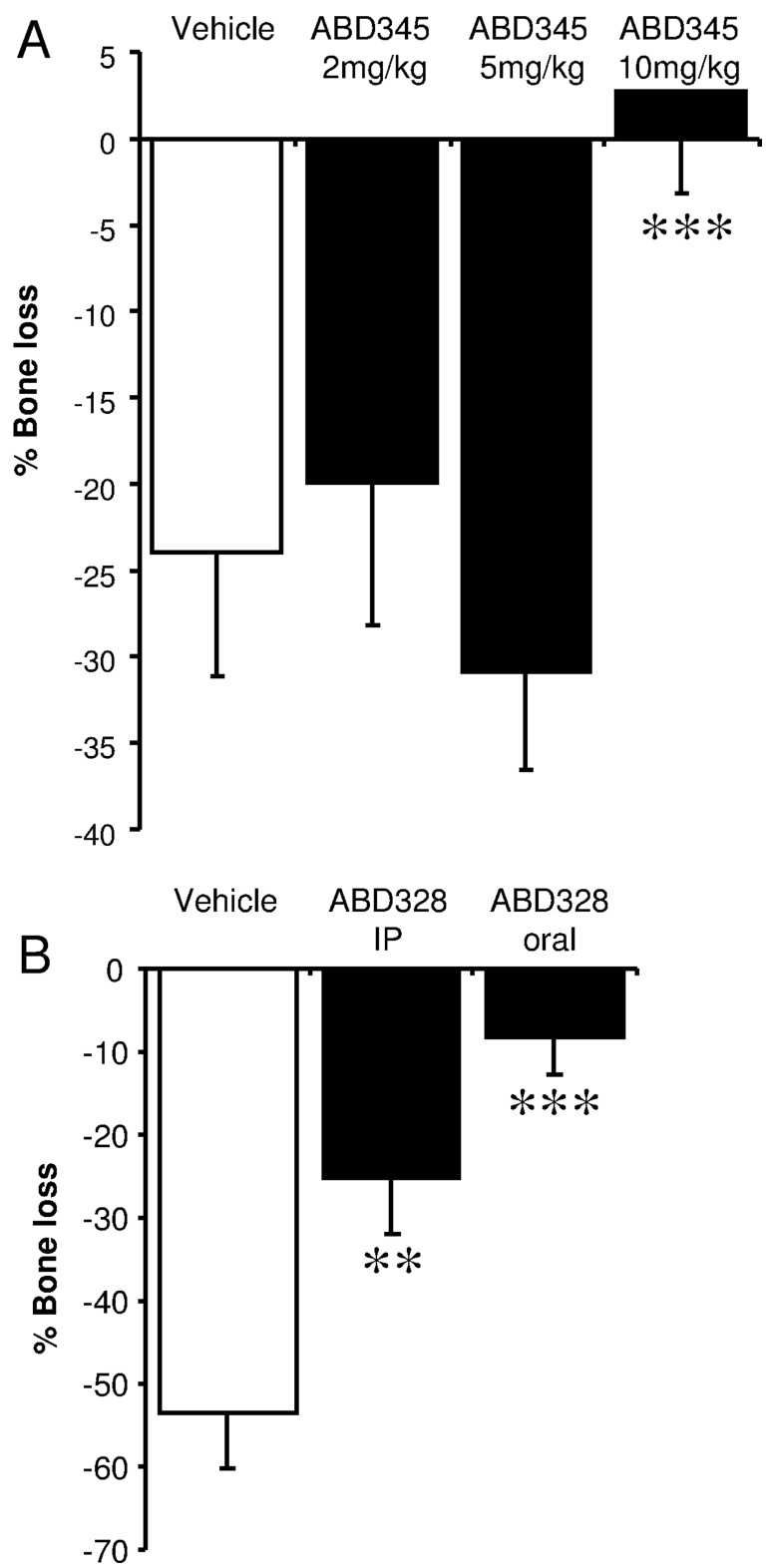

Figure 7 ABD328 and ABD345 inhibit inflammation-induced bone loss. Trabecular bone volume of the proximal tibia was measure by $\mu \mathrm{CT}$ analysis. Data are expressed as difference from control animals. collagen induced arthritis (CIA) leads to a pronounced decrease in trabecular bone volume, which is inhibited by $10 \mathrm{mg} / \mathrm{kg} / \mathrm{day}$ ABD345 (A) and ABD328, both by intraperitoneal injection and oral dosing (B). Values are averages $\pm \mathrm{SD}(\mathrm{N}=8)$. Data were analysed by analysis of variance (ANOVA). ${ }^{* *} \mathrm{p}<0.01 ;{ }^{* * *} \mathrm{p}<0.001$. high levels in early disease predict progression to erosive disease. ${ }^{17}$ The inhibitory effect of the compounds on TNF-induced JNK and ERK activation may therefore enhance the anti-inflammatory effects of the compounds.

The compounds did not affect macrophage viability, even at much higher concentrations than those necessary to inhibit osteoclast formation or cytokine-induced signalling. We have previously shown that these compounds also have no effect on osteoblast viability or function, ${ }^{16}$ indicating that the effects of the compounds are not due to general toxicity. In support of this, visual inspection of drug treated animals at time of death did not show any evidence of toxicity.

Although the precise molecular target for these compounds remains unclear, the evidence presented here suggests that they may inhibit NFאB and MAPK signalling by inhibiting recruitment or activation of signalling molecules common to both the TNF and RANK receptors. Signalling downstream of the TNF and RANK receptors involves the interaction of a large number of signalling molecules. One of the first steps in both RANKL and TNF signalling is recruitment of TRAF molecules to the RANK and TNF receptors. However, TNF-induced signalling is mostly dependent on TRAF-2 and -5, whereas RANKL-induced signalling is mostly mediated by TRAF- 6 . As the binding motif for TRAF- 6 is different to that for TRAF-2 and TRAF-5, ${ }^{11} 18$ it is unlikely that the compounds directly inhibit the recruitment of TRAFs to the receptors. It is more likely that the compounds interact with other members of the signalling complexes involved in RANKL and TNF signalling, leading to destabilisation of these complexes. One possible target that is common to both TNF- and RANK-signalling is the kinase TAK1, which is important for activation of both the NFKB and MAPK pathways. However, in the RANK signalling complex, TAK1 is recruited through TAB1 and TAB2, ${ }^{19}$ whereas in TNF signalling TAK 1 is recruited by RIP ${ }^{20}$ and TAB 1 and TAB 2 are not necessary for signalling activity. ${ }^{21}$ Another possible target for the compounds could be ubiquitination which has been shown to be important in both TNF- and RANKL-induced signalling. ${ }^{22} 23$

Inflammatory diseases such as rheumatoid arthritis are also characterised by osteoclastic bone destruction, ${ }^{24}$ and rheumatoid arthritis is associated with increased risk of fracture. ${ }^{25}$ Steroids such as prednisolone or dexamethasone are widely used to treat inflammatory disorders such as rheumatoid arthritis. However, although very potent inhibitors of inflammation, these drugs can cause steroid-induced osteoporosis, ${ }^{26}$ further increasing the fracture risk. The novel ABD compounds described here, in contrast, couple anti-inflammatory effects with bone protective effects.

Neutralising antibodies to TNF or decoy TNF-receptors have been shown to be highly effective treatments for inflammatory diseases such as rheumatoid arthritis, ${ }^{27}$ psoriatic arthritis ${ }^{28}$ and ankylosing spondylitis, ${ }^{29} 30$ and there is evidence that anti-TNF therapy slows the rate of bone erosions and systemic bone loss in rheumatoid arthritis. ${ }^{24} 3132$ However, a recent review of the available data suggests that the antiresorptive effects of anti-TNF therapy are related to control of disease activity rather than direct suppression of osteoclast activity. ${ }^{33}$ Furthermore, a study by Chopin $e t \mathrm{al}^{34}$ showed that although anti-TNF therapy suppressed bone resorption markers in the early phases of the treatment, bone resorption levels had returned to baseline by 1 year of treatment. A study by Vis et al has shown that although anti-TNF treatment halts further bone loss in the spine and hip, bone loss in the hand does progress ${ }^{35}$ and a recent study of fracture risk in patients with autoimmune disease showed no decrease in fracture risk in patients treated with anti-TNF ${ }^{36}$ compared with those on conventional therapy. Preclinical 
studies have also shown that blockade of RANKL-induced RANK signalling is required for full protection against inflammation-induced bone destruction in vivo. ${ }^{3} 63738$ In the present study, the antiresorptive effects of the ABD compounds could be due either to suppression of the inflammation or an anti-RANKL effect. Furthermore, the duration of the CIA experiments is too short to draw solid conclusions about the compounds' long term protection against inflammatory bone loss. However, the direct effects of the ABD compounds on osteoclast formation and activity reported earlier ${ }^{8} 910$ suggest that these compounds may give additional protection from inflammatory bone loss.

To our knowledge, the ABD compounds are currently the only dual inhibitors of both TNF- and RANKL-induced signalling, and therefore these new compounds show promise as treatments for inflammation and bone destruction associated with chronic inflammatory conditions such as rheumatoid arthritis. As small molecules, the ABD compounds may be cost-effective alternatives to treatment with biologics such as anti-TNF. Furthermore, as one of the compounds, ABD328, showed oral activity, the administration of these compounds may avoid the problems and costs associated with compounds administered by injection or intravenous drip.

Contributors EC performed experiments and analysed data. IRG designed experiments, designed and synthesised the novel compounds and co-wrote the paper. PM provided essential materials and advice. LR performed the experiments. MG designed and performed experiments. SHR designed experiments and co-wrote the paper. RJvH designed and performed experiments, analysed data and wrote the paper.

Funding This work was supported in part by grants from Arthritis Research UK (grant numbers: 17362 and 18328), Scottish Enterprise and NesTech.

Competing interests SHR, IRG and RJvH are inventors on a patent for the use of the compounds as anti-inflammatory drugs, held by the University of Aberdeen.

Ethics approval All animal experiments were approved by the University of Edinburgh's ethics committee.

Provenance and peer review Not commissioned; externally peer reviewed.

\section{REFERENCES}

1 Li J, Sarosi I, Yan XQ, et al. RANK is the intrinsic hematopoietic cell surface receptor that controls osteoclastogenesis and regulation of bone mass and calcium metabolism. Proc Natl Acad Sci USA 2000;97:1566-71.

2 Gohda J, Akiyama T, Koga T, et al. RANK-mediated amplification of TRAF6 signaling leads to NFATc1 induction during osteoclastogenesis. EMBO J 2005;24:790-9.

3 Kong YY, Feige U, Sarosi I, et al. Activated T cells regulate bone loss and joint destruction in adjuvant arthritis through osteoprotegerin ligand. Nature 1999;402:304-9.

4 Lam J, Takeshita S, Barker JE, et al. TNF-alpha induces osteoclastogenesis by direct stimulation of macrophages exposed to permissive levels of RANK ligand. I Clin Invest 2000;106:1481-8.

5 Redlich K, Hayer S, Ricci R, et al. Osteoclasts are essential for TNF-alpha-mediated joint destruction. J Clin Invest 2002;110:1419-27.

6 Redlich K, Gortz B, Hayer S, et al. Repair of local bone erosions and reversal of systemic bone loss upon therapy with anti-tumor necrosis factor in combination with osteoprotegerin or parathyroid hormone in tumor necrosis factor-mediated arthritis. Am J Pathol 2004;164:543-55.

7 Jimi E, Aoki K, Saito H, et al. Selective inhibition of NF-kappa B blocks osteoclastogenesis and prevents inflammatory bone destruction in vivo. Nat Med 2004; 10:617-24.

8 van't Hof RJ, Idris Al, Ridge SA, et al. Identification of biphenylcarboxylic acid derivatives as a novel class of bone resorption inhibitors. J Bone Miner Res 2004; 19:1651-60.

9 Idris Al, Mrak E, Greig I, et al. ABD56 causes osteoclast apoptosis by inhibiting the NFkappaB and ERK pathways. Biochem Biophys Res Commun 2008;371:94-8.

10 Idris Al, Coste E, Greig IR, et al. The biphenyl-carboxylate derivative ABD328 is a novel orally active antiresorptive agent. Calcif Tissue Int 2010;87:525-32.

11 Dempsey PW, Doyle SE, He JQ, et al. The signaling adaptors and pathways activated by TNF superfamily. Cytokine Growth Factor Rev 2003;14:193-209.

12 Hayden MS, Ghosh S. Shared principles in NF-kappaB signaling. Cell 2008;132:344-62.
13 Greig IR, Idris Al, Ralston SH, et al. Development and characterization of biphenylsulfonamides as novel inhibitors of bone resorption. J Med Chem 2006:49:7487-92.

14 Greig IR, Coste E, Ralston SH, et al. Discovery of biphenylketones as dual modulators of inflammation and bone loss. Bioorg Med Chem Lett 2010;20:5548-51.

15 Idris Al, Krishnan M, Simic $P$, et al. Small molecule inhibitors of IkappaB kinase signaling inhibit osteoclast formation in vitro and prevent ovariectomy-induced bone loss in vivo. FASEB J 2010;24:4545-55.

16 Idris Al, Greig IR, Bassonga-Landao $E$, et al. Identification of novel biphenyl carboxylic acid derivatives as novel antiresorptive agents that do not impair parathyroid hormone-induced bone formation. Endocrinology 2009:150:5-13.

17 de Launay D, van de Sande MG, de Hair MJ, et al. Selective involvement of ERK and JNK mitogen-activated protein kinases in early rheumatoid arthritis (1987 ACR criteria compared to 2010 ACR/EULAR criteria): a prospective study aimed at identification of diagnostic and prognostic biomarkers as well as therapeutic targets. Ann Rheum Dis 2012;71:415-23.

18 Darnay BG, Ni J, Moore PA, et al. Activation of NF-kappaB by RANK requires tumor necrosis factor receptor-associated factor (TRAF) 6 and NF-kappaB-inducing kinase. Identification of a novel TRAF6 interaction motif. J Biol Chem 1999:274:7724-31.

19 Feng $X$. Regulatory roles and molecular signaling of TNF family members in osteoclasts. Gene 2005;350:1-13.

20 Blonska $\mathrm{M}$, Shambharkar PB, Kobayashi $\mathrm{M}$, et al. TAK1 is recruited to the tumor necrosis factor-alpha (TNF-alpha) receptor 1 complex in a receptor-interacting protein (RIP)-dependent manner and cooperates with MEKK3 leading to NF-kappaB activation. J Biol Chem 2005;280:43056-63.

21 Shim JH, Xiao C, Paschal AE, et al. TAK1, but not TAB1 or TAB2, plays an essential role in multiple signaling pathways in vivo. Genes Dev 2005;19:2668-81.

22 Lamothe B, Webster WK, Gopinathan A, et al. TRAF6 ubiquitin ligase is essential for RANKL signaling and osteoclast differentiation. Biochem Biophys Res Commun 2007:359:1044-9.

23 Walczak H. TNF and ubiquitin at the crossroads of gene activation, cell death, inflammation, and cancer. Immunol Rev 2011;244:9-28.

24 Roux C. Osteoporosis in inflammatory joint diseases. Osteoporos Int 2011:22:421-33.

25 van Staa TP, Geusens P, Bijlsma JW, et al. Clinical assessment of the long-term risk of fracture in patients with rheumatoid arthritis. Arthritis Rheum 2006;54:3104-12.

26 van Staa TP, Leufkens HG, Cooper C. The epidemiology of corticosteroid-induced osteoporosis: a meta-analysis. Osteoporos Int 2002;13:777-87.

27 Feldmann M, Brennan FM, Paleolog E, et al. Anti-TNFalpha therapy of rheumatoid arthritis: what can we learn about chronic disease? Novartis Found Symp 2004:256:53-69.

28 Ogilvie AL, Antoni C, Dechant C, et al. Treatment of psoriatic arthritis with antitumour necrosis factor-alpha antibody clears skin lesions of psoriasis resistant to treatment with methotrexate. Br J Dermatol 2001;144:587-9.

29 Braun J, Baraliakos X, Brandt J, et al. Persistent clinical response to the anti-TNF-alpha antibody infliximab in patients with ankylosing spondylitis over 3 years. Rheumatology (Oxford) 2005;44:670-6.

30 Sieper J, van der HD, Dougados $M$, et al. Early response to adalimumab predicts long-term remission through 5 years of treatment in patients with ankylosing spondylitis. Ann Rheum Dis 2012;71:700-6.

31 Seriolo B, Paolino S, Sulli A, et al. Bone metabolism changes during anti-TNF-alpha therapy in patients with active rheumatoid arthritis. Ann NY Acad Sci 2006;1069:420-7.

32 Wijbrandts CA, Klaasen R, Dijkgraaf MG, et al. Bone mineral density in rheumatoid arthritis patients 1 year after adalimumab therapy: arrest of bone loss. Ann Rheum Dis 2009;68:373-6.

33 Kawai VK, Stein CM, Perrien DS, et al. Effects of anti-tumor necrosis factor alpha agents on bone. Curr Opin Rheumatol 2012;24:576-85.

34 Chopin F, Garnero P, le Henanff A, et al. Long-term effects of infliximab on bone and cartilage turnover markers in patients with rheumatoid arthritis. Ann Rheum Dis 2008:67:353-7.

35 Vis M, Havaardsholm EA, Haugeberg G, et al. Evaluation of bone mineral density, bone metabolism, osteoprotegerin and receptor activator of the NFkappaB ligand serum levels during treatment with infliximab in patients with rheumatoid arthritis Ann Rheum Dis 2006:65:1495-9.

36 Kawai VK, Grijalva CG, Arbogast PG, et al. Initiation of Tumor Necrosis Factor Alpha (TNFalpha) antagonists and risk of fractures in patients with selected rheumatic and autoimmune diseases. Arthritis Care Res (Hoboken) 2013:65:1085-94.

37 Redlich K, Hayer S, Maier A, et al. Tumor necrosis factor alpha-mediated joint destruction is inhibited by targeting osteoclasts with osteoprotegerin. Arthritis Rheum 2002:46:785-92.

38 Schett G, Redlich K, Hayer S, et al. Osteoprotegerin protects against generalized bone loss in tumor necrosis factor-transgenic mice. Arthritis Rheum 2003;48:2042-51. 